International Journal of Wireless \& Mobile Networks (IJWMN) Vol. 3, No. 6, December 2011

\title{
Power Aware QoS Multipath Routing Protocol FOR DiSASTER RECOVERY NETWORKS
}

\author{
S.Santhi ${ }^{1}$, Dr.G.Sudha Sadasivam ${ }^{2}$ \\ ${ }^{1}$ Department of Information Technology,Dr.NGP Institute of \\ Technology,Coimbatore,India \\ shanthimathandyahoo.co.in \\ ${ }^{2}$ Department of Computer Science and Engineering,PSG College of \\ Technology,Coimbatore,India \\ sudhasadhasivameyahoo.co.in
}

\begin{abstract}
Mobile communication plays an important role in disaster recovery management during emergency situations. It is helpful in situations where the system has less robust and less flexible fixed infrastructure. The Disaster recovery management systems require timely interaction and coordination in order to save lives and property. Energy consumption in heterogeneous network is a major issue, whether they operate within a base station infrastructure, fixed network or in a free-standing Mobile Ad Hoc Network (MANET). The lifetime of network will be improved by suitably reducing the requirement of power for connections. There is a challenge to provide Quality of Service $(Q o S)$ solutions to wired cum wireless domains and maintain end-to-end QoS in ad hoc network. In this paper we propose a new protocol Power Aware QoS Multipath Routing protocol (PAQMR) for disaster recovery network. This protocol is the enhanced protocol of Ad-Hoc On Demand Multipath Distance Vector protocol (AOMDV). This routing protocol is used to avoid the loop formation in network so that it reduces congestion in the channel. The Network Simulator (NS-2.34) tool is utilized to measure the performance of AODV, AOMDV and $P A Q M R$ protocols in hybrid environment. The metrics for the simulation are energy consumption, average end to end delay and packet delivery ratio by varying the traffic load and pause time in the network. The results shows that the proposed protocol minimize the power, delay, congestion and maximize the packet delivery ratio.
\end{abstract}

\section{KEYWORDS}

PAQMR, AODV, AOMDV, Quality of Service, Average End to End delay, MANET, Packet Delivery Ratio.

\section{INTRODUCTION}

The communication networks and other infrastructures got destroyed during natural and manmade disasters. These may lead to network components failure, physical destruction of supporting network infrastructure and these two together may cause network congestion. During this emergency situation the wireless ad-hoc network plays an important role to save human life and properties. The main goal of our framework is to create a new topology and protocol by which the various systems of the network connected and power will be saved, in order to be able to continue transmitting the important information. A heterogeneous network consists of collection of wired as well as wireless nodes. This paper develops a new protocol for energy consumption. By examining energy consumption, the important factors are the protocol behaviors and network-layer issues in the ad hoc environment. The challenging task of routing in heterogeneous is to provide expected Quality of Service (QoS). The main goal of a QoS multipath routing protocol is to identify loop free paths from the source to the destination with the available resources to meet the QoS requirements of the desired service. The advantages are 
better network utilization, network resilience to failures and efficient usage of bandwidth. Multipath routing separates the traffic among different paths to minimize congestion in terms of multiple alternative paths through a network which can provide a variety of benefits such as minimize delay as well as congestion, maximize bandwidth and improved security.

Ad hoc Routing protocols is classified into two types such as proactive and reactive. The table-driven routing protocol is proactive, it is worked on distance vector based or link state based routing strategies. The drawback of this algorithm is the frequent table updation required which consumes large amount of memory, bandwidth and power [1]. But, in the reactive routing protocol, each node does not need to maintain the routing table. When a source node is ready to send data, it initiates the route discovery procedure and maintains its routes only. The reactive routing protocol minimizes the routing overhead and also called on-demand approach.

The AODV [2] protocol based on the reactive routing discovery uses three different kinds of messages: Route request (RREQ), Route Reply (RREP) and Route Error (RERR). In addition, destination sequence numbers are used to ensure loop freedom at all times. In AODV, each source node finds a new route by the limited flooding of RREQ and obtains a route to its destination through RREP. The AOMDV [3] protocol is the extension of AODV routing protocol, in which the source node keeps several different alternative routes from multiple RREPs. The static route selection is used in AOMDV, it cannot handle the dynamic change of the network due to severe congestion caused by biased traffic.

In this paper new modified version of AOMDV routing protocol is proposed and also the performance of different routing protocols are evaluated in terms of the power consumption, average end-to-end delay, packet delivery ratio of the network are calculated.

This paper is organized as follows: Section 2 deals with the mathematical model for energy constraint in heterogeneous network and Section 3 briefly details different routing protocols for Heterogeneous Network. Section 4 discusses the Simulation model, Mobility Model and Traffic Model for wired cum wireless network. Section 5 deals with Result Analysis, in Section 6, Conclusions are outlined.

\section{RELATED WORKS}

Quality of Service provisioning is one of the important feature for Mobile Ad hoc Networks (MANET), Also energy conservation is a major issue in the ad hoc networks for saving network life time with limited battery power. The main goal of a QoS multipath routing protocol is to identify loop free paths from the source to the destination with the available resources to meet the QoS requirements of the desired service. The advantages are better network utilization, network resilience to failures and efficient usage of bandwidth. In [4] proposed an energy management model for reduce energy consumption in MANET and a new routing protocol with joint function considering both transmission power and residual energy of mobile nodes. Multipath Routing with Load Balancing QoS in Ad hoc Network [5] gives new protocol for Ad Hoc routing. It deals with only delay that does not satisfy the bandwidth and energy constraint. But in our paper we consider bandwidth and energy constraints for selecting best path from source to destination node. The energy stable Quality of Service routing technique [8] determine bandwidth constrained paths and energy stability for local reconstruction of the routes to avoid packet loss. The network lifetime is enhanced and the overall efficiency of packet delivery is also improved in this paper. Here a prior estimation of the bandwidth and admission control to ensure bandwidth availability between wireless links is required to ensure the performance of the protocol. A lot of research work on energy efficient routing in mobile ad hoc networks has been done in [9-16]. The above protocols are based on homogeneous nodes and hence are not suitable for hybrid nodes with heterogeneous link characteristics. In [6] and [7] discuss the techniques for hybrid network and their implementation using DSR, AODV and AOMDV 
International Journal of Wireless \& Mobile Networks (IJWMN) Vol. 3, No. 6, December 2011

protocols. But our proposed protocol differs from other multipath routing protocols because the protocol has been developed for hybrid nodes with heterogeneous link characteristics also consider QoS metrics for energy consumption.

\section{Mathematical Model}

In this work we model energy for heterogeneous network, It is a collection of wired, wireless and mobile networks. The convergence of this network is the success of the next generation networking. It has some challenge like

- Maximize network resources utilization, and Minimize operational costs, delay and bandwidth on all the types of wired-wireless-mobile networks

- Mix the QoS associated with Fixed, Mobile and Core networks.

Consider a heterogeneous network that is represented by a directed graph $\mathrm{G}=(\mathrm{V}, \mathrm{E})$. Where $\mathrm{V}$ is the set of wired and wireless nodes and $\mathrm{E}$ is the set of links between different networks. Each $\operatorname{link}(\mathrm{i}, \mathrm{j}) € \mathrm{E}$ is associated with a primary cost parameter $\mathrm{c}(\mathrm{i}, \mathrm{j})$ and energy $\mathrm{e}_{\mathrm{ij}}$ is additive QoS parameter for multiple path $\mathrm{P}=1,2, \ldots . . \mathrm{p}$. The problem is to find a shortest path from source to destination and minimize energy.

(i) A Heterogeneous network is a collection of wired, wireless and mobile networks. It is denoted as

$$
\mathrm{N}=(\mathrm{N} 1, \mathrm{~N} 2, \mathrm{~N} 3 \ldots . . \mathrm{Nn}) \text {. }
$$

$\mathrm{N}$ is the heterogenous network. $\mathrm{N} 1, \mathrm{~N} 2 \ldots . \mathrm{Nn}$ may be wired, wireless and mobile node.

(ii) Multiple path is denoted as P.

$$
\mathrm{P}=\mathrm{P} 1, \mathrm{P} 2 \ldots \ldots \mathrm{Pn}
$$

$\mathrm{n}$ is a number of paths. Message passed over mulitiple paths. Load is shared between various links between source to destination

(iii) The set $\mathrm{Ri}=\mathrm{R} 1, \mathrm{R} 2, \mathrm{R} 3 \ldots . . \mathrm{Rn}$ represents the distribution of the load across the set of the resources. Total traffic sent from the source is $\mathrm{R}=\sum \mathrm{Ri}$

\subsection{Power Aware Model}

This model is discussed below.

The Energy is calculated by using this formula.

Energy $=$ Power $*$ Time

The energy consumption is measured by the transmitting power or receiving power multiply the transmitted time.

$$
\mathrm{Pt}=\frac{(8 * \text { Packet size })}{\text { Bandwidth }}
$$

The Transmitting Energy $\mathrm{E}_{\mathrm{tx}}$ is defined as

$\mathrm{E}_{\mathrm{tx}}=\mathrm{P}_{\mathrm{tx}} * \mathrm{P}_{\mathrm{t}}$

The Receiving Energy $\mathrm{E}_{\mathrm{rx}}$ is defined as 
International Journal of Wireless \& Mobile Networks (IJWMN) Vol. 3, No. 6, December 2011

$\mathrm{E}_{\mathrm{rx}}=\mathrm{P}_{\mathrm{rx}} * \mathrm{P}_{\mathrm{t}}$

Energy consumption of a node after time $t$ is calculated using the following equation.

$\mathrm{E}_{\mathrm{con}}(\mathrm{t})=\mathrm{N}_{\mathrm{t}} * \mathrm{C}_{1}+\mathrm{N}_{\mathrm{r}} * \mathrm{C}_{2}$

Where

$\mathrm{E}_{\text {con }}(\mathrm{t})$, energy consumed by a node after time $\mathrm{t}$.

$\mathrm{N}_{\mathrm{t}}$, no. of packets transmitted by the node after time $\mathrm{t}$.

$\mathrm{N}_{\mathrm{r}}$, no. of packets received by the node after time t.

$\mathrm{C}_{1}$ and $\mathrm{C}_{2}$ are constant factors having a value between 0 and 1 .

Let $\mathrm{E}$ be the initial energy of a node and the residual energy $\mathrm{E}_{\mathrm{Res}}$ of a node at time $\mathrm{t}$, can be calculated by using the formula

$\mathrm{E}_{\text {Res }}=\mathrm{E}-\mathrm{E}_{\mathrm{con}}(\mathrm{t})$

Total energy consumption of all Nodes is measured as the summation of all node's residual energy plus the product of initial energy and number of nodes.

$\mathrm{TE}_{\mathrm{con}}=\mathrm{N} *$ Initial Energy $-\mathrm{E}_{\mathrm{Res}}$

Here $\mathrm{N}$ is denotes as the number of nodes used in the network.

Table 1. Notation for the Mathematical Model

\begin{tabular}{|c|l|}
\hline Symbol & Description \\
\hline $\mathrm{P}_{\mathrm{t}}$ & Transmitted Time \\
\hline $\mathrm{E}_{\mathrm{tx}}$ & Transmitted Energy \\
\hline $\mathrm{E}_{\mathrm{rx}}$ & Received Energy \\
\hline $\mathrm{P}_{\mathrm{tx}}$ & Transmitted Power \\
\hline $\mathrm{P}_{\mathrm{rx}}$ & Received Power \\
\hline $\mathrm{E}_{\mathrm{Int}}$ & Initial Energy \\
\hline $\mathrm{E}_{\mathrm{Res}}$ & Residual Energy \\
\hline $\mathrm{E}_{\mathrm{Con}}$ & Consumed Energy \\
\hline $\mathrm{TE}_{\mathrm{Con}}$ & Total Consumed Energy \\
\hline
\end{tabular}

\section{Protocols for Heterogeneous Network}

\subsection{Hierarchical Addressing}

The network hierarchy is arrangement of the nodes in the network. OSPF or IS-IS are examples of hierarchic protocols, these are link-state protocols in connection oriented network. This mechanism is helpful for scalability and reduce the amount of traffic inside network. The flat networks use only two levels of address which is not support scalability. 
The network is divided into three levels of address, they are domain, cluster and nodes. If the routing protocol uses hierarchy techniques then group of nodes use unique addressable entity from the top of the hierarchy. The three main important advantages originated from this mechanism are the decline in the number of routing messages needed to converge, the reduction of the size on the route table (reducing the memory needed) and also the reduction of the convergence time.

The hierarchy scheme used to settle the direction of the information flow in all area. OSPF has contact to all areas with the backbone area thro hierarchical addressing.

\subsection{Ad-hoc On-Demand Distance Vector Routing Protocol (AODV)}

The properties Dynamic Source Routing (DSR) and Destination Sequenced Distance Vectoring (DSDV) are combined into Adhoc On Demand Distance Vector routing algorithm. The routediscovery and route maintenance of DSR and hop- by-hop routing, sequence numbers and beacons of DSDV are taken in this protocol.

\subsection{Ad-hoc On-Demand Multi-path Distance Vector Routing Protocol (AOMDV)}

Ad hoc On-demand Multipath Distance Vector (AOMDV) is the enhanced version of AODV protocol, it belongs to on demand and reactive routing protocol of ad-hoc wireless networks. The main goal is to compute multiple loop-free and link-disjoint paths between source and destination pair. The merit of AOMDV is estimated in terms of increased packet delivery ratio, throughput and reduced average end-to-end delay and normalized control overhead. The average end to end delay is reduced by introducing multiple loop free paths in this scheme. In multiple routes, the destination contains list of the next-hops along with the corresponding hop counts in routing table entries. Suppose all the next hops have the same sequence number. The advertised hop count is defined as the maximum hop count for all the paths. Route advertisement sends to destination by using this hop count value. If any duplicate route advertisement received by a node then it forwards the packet thro alternate path to the destination. The loop freedom is ensured by selecting the alternate path for destination on the basis of the hop count value of path is less than the advertised hop count for that destination. The destination node sorted all the paths by maximum hop count value. The best paths are selected and data forwarded through this paths. AOMDV may follows node-disjoint or linkdisjoint routes. In node-disjoint routes, duplicate RREQs can not immediately rejected. The RREQ and RREP pair arrives through different neighbour of the source in a node-disjoint path. During route discovery, the source node broadcasts a ROUTE REQUEST packet that is broadcasted throughout the network. In contrast to AODV, each recipient node creates multiple reverse routes while processing the ROUTE REQUEST packets that are received from multiple neighbours.

\subsection{Power Aware QoS Multipath Routing protocol (PAQMR)}

In PAQMR a feasible path is searched out which satisfies the bandwidth and energy constraint. In contrast to the flooding based algorithms, PAQMR search only a small number of paths, this limits the routing overheads. In order to maximize the chance of finding a feasible path, the information is collectively utilized to make hop by hop selection. This protocol does not consider the QoS requirement only but also considers the optimality of the routing path in terms of bandwidth and energy efficiency. If a specific QoS request is not being asked by a user then high energy paths are chosen by PAQMR in order to improve the overall network lifetime. In case of PAQMR a simple energy consumption model has been used to calculate the energy values at different times. This new scheme has three phases 


\subsubsection{Route Discovery}

In route discovery procedure, the Power Aware QoS Multipath Routing protocol (PAQMR) builds a route between source to destination using a route request and route reply query cycle. When a source node wants to send a packet to destination for which it does not already have a route, it forward a route request (RREQ) packet to all the neighbours across the network. The performance of PAQMR is improved by adding QoS parameters in RREQ packet, two additional fields are added in the RREQ header information such as bandwidth and energy constraints.

The extended Route Request packet of PAQMR is shown in figure 1.

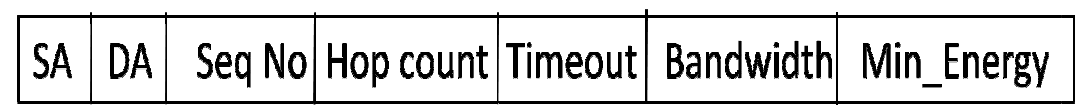

Figure 1. Extended Route Request Message

In PAQMR routing discovery process, the source node in the network sends the extended Route Request (RREQ) message to the destination node through number of intermediate nodes. The data transmission in wireless network can be directly within one hop or through number of intermediate nodes. The extended Route Request (RREQ) message contains the source and destination node IP address, Advertised Hop count value, Timeout value, Bandwidth of the link (Bandwidth) and Minimum Energy value. The computed bandwidth and minimal nodal energy is greater than the threshold value of bandwidth and energy then only the RREQ message forward to the next neighbor node otherwise it discarded. When the RREQ message arrive at next node , the bandwidth and minimal nodal energy is updated into the route list entries. The structure of routing table entries for AOMDV and PAQMR are given in Table 2.

Table 2. Routing table entries for AOMDV and PAQMR

\begin{tabular}{|c|c|}
\hline AOMDV & PAQMR \\
\hline Destination Address & Destination Address \\
\hline Sequence Number & Sequence Number \\
\hline Advertised Hopcount & Advertised Hopcount \\
\hline $\begin{array}{l}\text { Route List } \\
\left(\text { nexthop }_{1}, \text { hopcount }_{1} \ldots\right) \\
\cdot \\
\cdot \\
\left(\text { nexthop }_{n}, \text {,hopcount }_{n} \ldots .\right)\end{array}$ & $\begin{array}{l}\text { Route List } \\
\text { (nexthop }_{1}, \text {,hopcount }_{1}, \text { Bandwidth }_{1} \text {, } \\
\text { Min_energy } y_{1} \ldots \text { ) } \\
\quad \cdot \\
\cdot \\
\text { (nexthop } \text {,hopcount }_{n}, \text { Bandwidth }_{\mathrm{n}} \\
\text { Min_energy } \mathrm{y}_{\mathrm{n}} \ldots \text { ) }\end{array}$ \\
\hline Time out & Time out \\
\hline
\end{tabular}

At the initial stage the Source node's initial energy is entered into min_energy field, The residual energy is computed at every node in the network. This residual energy is compared with min_energy field of RREQ packet. If this value is less than the min_energy field, then it replaced by residual energy. While selecting the best path, the min_energy should be kept as the lowest among all the nodes in this route. The reverse route to source node is set same as AOMDV protocol. Once the Route Request (RREQ) packet is received by the destination node, the node will produce Route Reply (RREP) packet and send back to the source node. RREP 
packet is also included two additional fields Bandwidth and min_energy, the RREP packet records the routing information from the source to destination. The duplicate packet ID is received by the destination node, then it responds with a maximum of 3 RREP packets to the source node.

The following algorithm illustrates the Route Discovery process.

\section{Algorithm: Route Discovery}

\section{Step 1. Source node S}

a) Creates the RREQ packet with field values set as $\mathrm{SA}=\mathrm{S}, \mathrm{DA}=\mathrm{D}$, Seq.No=I, TTL $=\mathrm{T}$, Hops $=\mathrm{H}, \mathrm{BW}=0$,Min_energy = Initial energy;

b) Broad cast the RREQ packet to next neighbor node whose $\mathrm{BW}_{\mathrm{th}}>=\mathrm{BW}$.

Step 2. If the intermediate node will receive the RREQ packet

a) The Min_energy field in RREQ is updated by initial energy

b) Forward the RREQ packet to node 2

c) Calculate Node's Residual energy

d) This Residual Energy value is compared with Min_energy value in Routing table. The route is selected on the basis of Min_energy $>=$ Renergy and $\mathrm{BW}_{\mathrm{th}}>=\mathrm{BW}$. Otherwise the link between Node1 and Node2 is unavailable.

Step 3. If the node receiving the RREQ packet in $\mathrm{D}$, then the node $\mathrm{D}$

a) Generates the RREP packet for unicasting to source. The bandwidth field of the RREP packet is updated with the cumulative bandwidth of the path and Energy field should be updated by cumulative Energy.

b) D unicasts all the node disjoint paths back to the source node $\mathrm{S}$.

\subsubsection{Route Selection}

When the RREQ receives at the neighbour node, it forwards a RREP packet back to the source. Otherwise, it rebroadcasts the RREQ. If they may receive a processed RREQ, they discard the RREQ and do not forward it. If RREQ of multiple paths are received at source node, it stored by the hop count value. In AOMDV the route is selected on the basis of minimum number of hops. But the PAQMR protocol select the best path by sorting multi-route in descending order of nodal residual energy and bandwidth and the data packets are forwarded by using the maximal nodal residual energy.

\subsubsection{Route Maintenance}

In case the energy value is less than the threshold value Min_Energy then link is broken, an Route Error message (RERR) is sent back to the previous node to indicate the route breakage. If node receives this RERR message, it inform to the source node then it starts route discovery procedure again. 
International Journal of Wireless \& Mobile Networks (IJWMN) Vol. 3, No. 6, December 2011

\section{WirED CUM WireleSS Simulation}

\subsection{Simulation Environment}

Wired-cum-wireless allows simulation using both wired, wireless nodes and mobile IP integrated into the wireless model. Base station Node is created which plays the role of a gateway for the wired and wireless domains, it is responsible for delivering packets into and out of the wireless domain

Table 3. SIMULATION PARAMETERS

\begin{tabular}{|l|l|}
\hline Simulator & NS2 \\
\hline Routing Protocols & AODV,AOMDV,PAQMR \\
\hline Simulation Time(Sec) & 500 \\
\hline Simulation Area & $800 * 800$ \\
\hline Number of Wired Nodes & 10,20 \\
\hline Number of Mobile Nodes & $10,20,30,40,50,60,70$ \\
\hline Transmission Range(m) & $250 \mathrm{~m}$ \\
\hline Mobility Model & Random way point \\
\hline Maximum Speed & $10 \mathrm{~m} / \mathrm{sec}$ \\
\hline Pause Time & $10,20,30,40,50,60,70$ \\
\hline Connection Rate & 5 packets /sec \\
\hline Data Payload & 512 \\
\hline Traffic Type & CBR \\
\hline
\end{tabular}

\subsection{Traffic Model}

Random traffic connection of Continuous Bit Rate (CBR) can be setup between mobilenodes using a traffic scenario generator script. The source to destination pairs are spread randomly over the mobile and fixed network. The number of clusters and the packet sending rate in each cluster is varied by change the traffic load and pause time.

\subsection{Mobility Model}

The mobility model for the simulation is random waypoint model in a rectangular field. The field configurations used is $800 \mathrm{~m} * 800 \mathrm{~m}$ field with 10 to 70 nodes. Each packet starts its transmission from random location to random destination with randomly chosen speed. After the destination is reached it select another random destination after a pause. The pause time affects the relative speed of the mobile. 
International Journal of Wireless \& Mobile Networks (IJWMN) Vol. 3, No. 6, December 2011

\section{RESUlt ANALysiS}

The Network Simulator 2 (NS-2.34) tool is used to measure the energy efficiency and routing efficiency of AODV, AOMDV and PAQMR protocols using End to End delay, Packet delivery ratio and energy consumption against number of nodes and pause time.

\subsection{Packet Deliver Fraction}

Packet Delivery Fraction is defined as the ratio between total number of packets delivered with number of packets sent. The effect of pause time and number of nodes on packet delivery ratio is evaluated.

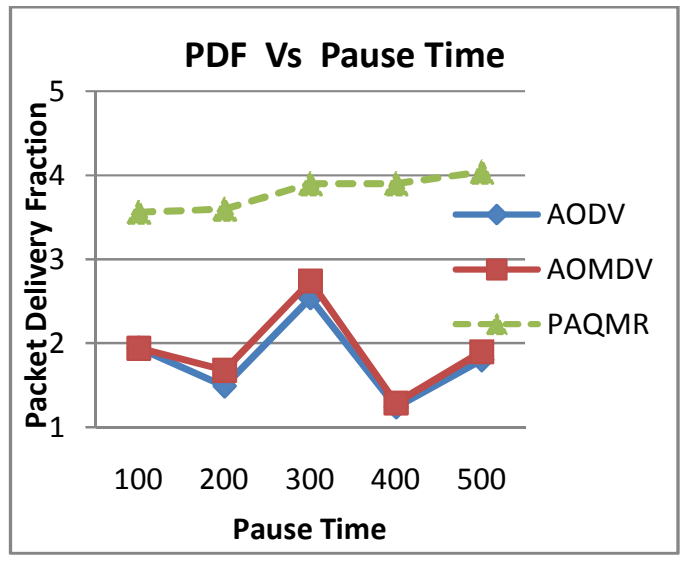

Figure 2. PDF Vs Pause Time

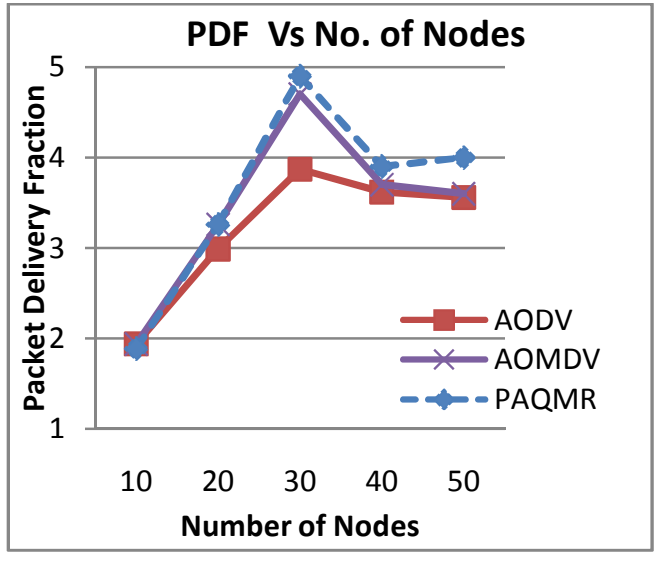

Figure 3. PDF Vs No. of Nodes

Figure 2 and 3 illustrate the packet delivery fraction against pause time and number of nodes. It shows that packet delivery ratio of PAQMR protocol is higher than other remaining two protocols.

\subsection{Energy Consumption}

The energy consumption of new protocol is lesser than AODV and AOMDV routing protocol. The total energy consumption is computed from initial energy and residual energy of all nodes connected in the network.

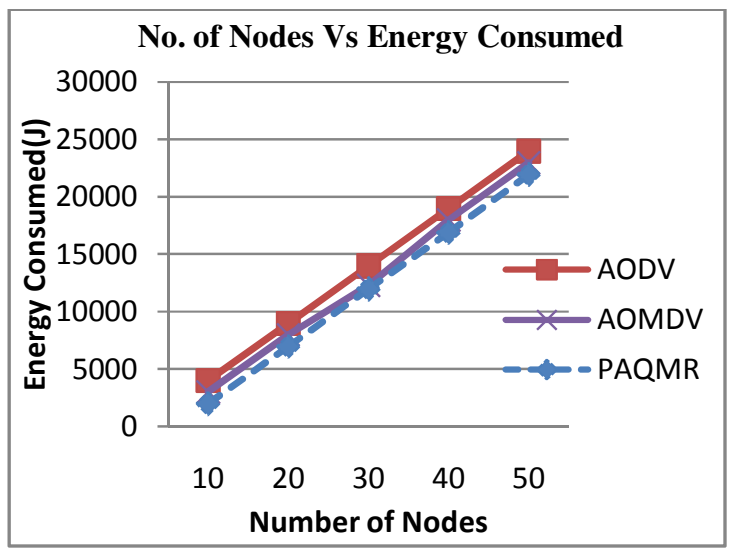

Figure 4 . Energy Consumed VS No. of Nodes 
In figure 4, 10 to 50 nodes network considered with a pause time of 100 seconds. It can be seen from the figure that in PAQMR, the consumed energy is reduced by including energy constraint during the path selection.

\subsection{End to End Delay}

The average end to end delay includes the queue delay and the propagation delay from the source to the destination.

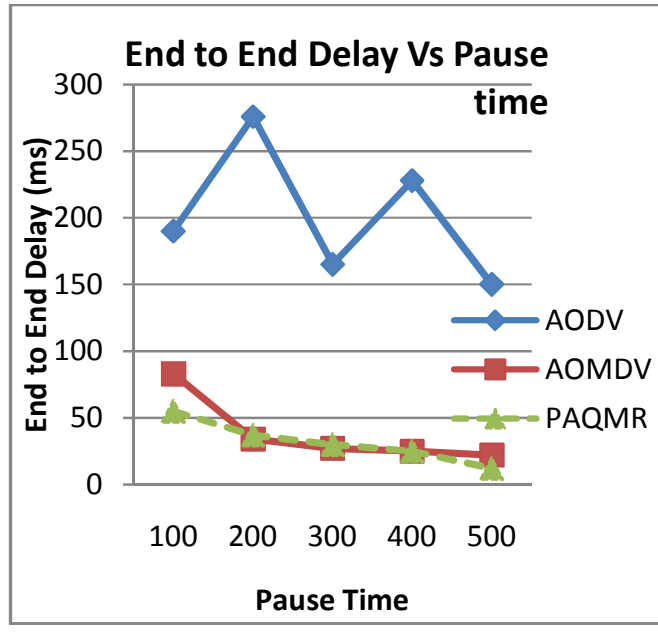

Figure 5. End to End Delay Vs Pause Time

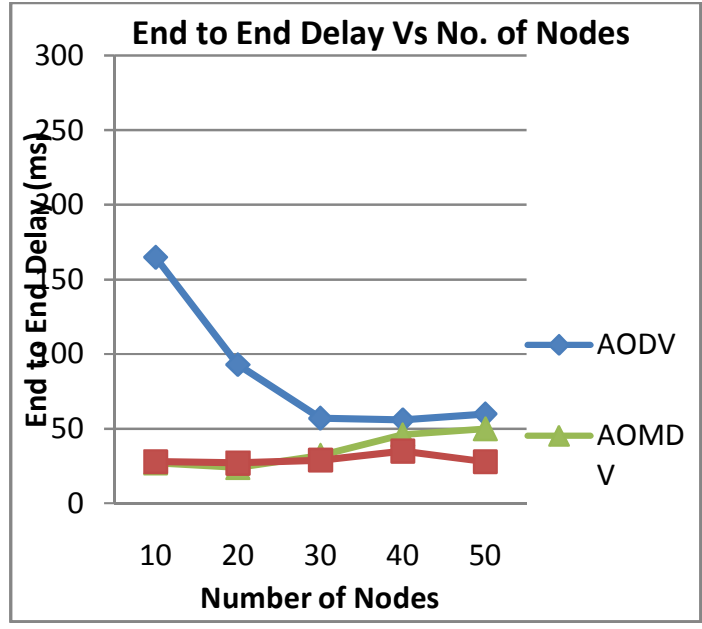

Figure 6.End to End Delay Vs No of Nodes

The multipath routing could effectively reduce the queue delay because the traffic is distributed among different routes and energy constraint is implemented in every node to select the optimal path. By varying the nodes from 10, 20 to 50, the average end to end delay measured against pause time and number of nodes in figure 5 and 6 . It seems that the delay of new protocol is reduced when comparing AODV and AOMDV protocols.

\section{CONCLUSION}

In this paper, we propose a new hybrid routing protocol for disaster recovery management. The Power Aware QoS Multipath Routing protocol(PAQMR) considers energy stability for route reconstruction to avoid packet loss. The goal of our scheme aims to find an optimal path in terms of bandwidth and energy-constrained applications. Performance evaluation has been done using NS2 simulator tool and comparison with AODV, AOMDV shows that our protocol can effectively reduce end to end delay and energy consumption while maintaining a good packet delivery ratio. The enhanced protocol has been developed for hybrid network with heterogeneous characteristics.

\section{REFERENCES}

[1] E. M. Royer and C. K. Toh, (1999) "A Review of Current Routing Protocols for Ad Hoc Mobile Wireless Networks", IEEE Personal Communications, Vol. 6, No. 2, pp 46-55.

[2] T.Bheemarjuna Reddy, I.Karthigeyan, B.S.Manoj and C.Siva Ram Murthy, (2006) "Quality of service provisioning in ad hoc wireless networks: a survey of issues and solutions", Elsevier B. V., Vol.4,No.3. 
International Journal of Wireless \& Mobile Networks (IJWMN) Vol. 3, No. 6, December 2011

[3] K.Mahesh, Marina, Samir R. Das (2001) "Ad hoc on-demand multipath distance vector routing", International Journal of Wireless Communications And Mobile Computing, Vol. 6,No.2 ,pp. 969988.

[4] Yu Wang, (2010) "Study on Energy Conservation in MANET", Journal of Networks, Vol. 5, No. 6, pp. 708-715.

[5] Mohamed Tekaya, Nabil Tabbane, Sami Tabbane, (2010) "Multipath Routing with Load Balancing and QoS in Ad hoc Network”, IJCSNS International Journal of Computer Science and Network Security, Vol. 10, pp. 280-286.

[6] Koushik Majumder,Subir kumar sarkar, (2010) “Analysis of QOS Parameters for DSDV and DSR in Hybrid Scenario", IEEE International Symposium on Electronic System Design (ISED 2010), India.

[7] S.Santhi, G.Sudha Sadhasivam, (2011) "Performance Analysis of Different QoS Routing Protocols to Minimize Power in Heterogeneous Network", Proceeding Of IEEE International Conference on PACC 2011, India.

[8] Fujian Qin, (2011) "QoS Topology Control with Energy Efficiency for MANET”, Journal of Convergence Information Technology, Vol. 6, No. 6,pp. 300-307.

[9] J.L.Lian, Li and X. Zhu, (2007) "A multiple QOS constraints routing protocol based on mobile predicting in ad hoc network", Proceedings of IEEE International Conference on Wireless Communications, Networking and Mobile Computing ,pp. 1608-1611.

[10] B.Malarkodi,S.K.Riyaz Hussain, and B.Venkataramani, (2010) "Performance Evaluation of AOMDV-PAMAC protocols for Ad Hoc Networks", World Academy of Science , Engineering and Technology, pp. 539-542.

[11] S.Vinay Rishiwal Verma and S. K. Bajpai, (2009) "QoS Based Power Aware Routing in MANETs" ,International Journal of Computer Theory and Engineering, Vol. 1, No. 1, pp. 47-54

[12] Laura Marie Feeney, (2001) “An Energy Consumption Model for Performance Analysis of Routing Protocols for Mobile Ad Hoc Networks", Mobile Networks and Applications 6, pp. 239-249.

[13] S. Singh, M Woo, and C S Raghavendra, (1998) "Power Aware Routing in Mobile Ad hoc Networks, ", ACM/IEEE Iinternational Conference on Mobile Computing and Networking, pp 181190.

[14] Rong Zheng and Robin Kravats, (2005) "On Demand Power Management for Adhoc Networks, " Journal of Adhoc Networks, Elsevier, Vol. 3, pp. 51-68.

[15] Jae Hwan Chang and Leandros Tassiulas, (2000) "Energy Conserving Routing in wireless adhoc network, " Proceeding in IEEE INFOCOMP, Tel Aviv, Israel, Vol. 1, pp 22-31.

[16] Feeney, L.M. Nilsson, M, (2001) "Investigating the Energy Consumption of a Wireless Network Interface in an Adhoc Network Environment ", Proceeding of Twentieth Annual Joint Conference of the IEEE Computer and Communications Societies ( INFOCOM 2001), AK, USA. Vol. 3, pp 15481557.

[17] Pascal Merindol and Jean-Jacques Pansiot and Stephane Cateloin, (2009) "Low Complexity Link State Multipath Routing”, IEEE/ACM Transactions on Networks, Brazil.

\section{Authors}

Ms S Santhi received the B.E. degree in Computer Science and Engineering from Institute of Road and Transport Technology, Erode, India in 1995, and M.E. degree in VLSI Design from Government College of Technology, Coimbatore, India in 2003. She is currently working towards the $\mathrm{PhD}$ degree in Computer Science and Engineering under Anna University of Technology, Coimbatore, India. She has published 10 papers in National and International conference and journal. Her research work is in Heterogeneous Network to improve the performance of network by Quality of Service parameters.

Dr G Sudha Sadasivam is working as a professor in CSE Department of PSG College of Technology. She has 20 years of teaching experience. She has published 5 books and 20 papers in referred journals. She has coordinated two AICTE-RPS projects in the areas of distributed computing. She is the coordinator of PSG-Yahoo research in grid and cloud computing and Xurmo Research in social networking
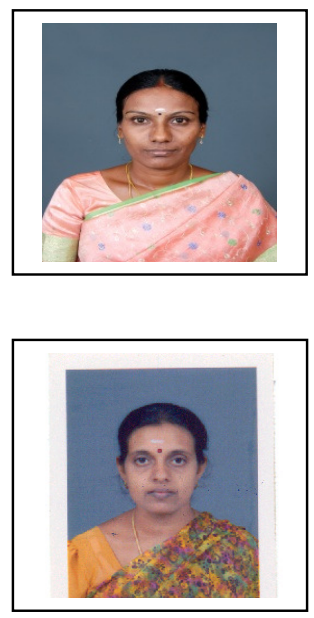